\title{
Software Tools for Energy Storage Valuation and Design
}

\author{
Tu A. Nguyen ${ }^{1}$ (D) Raymond H. Byrne ${ }^{1}$
}

Accepted: 9 May 2021 / Published online: 2 June 2021

(C) The Author(s) 2021

\begin{abstract}
Purpose of Review As the application space for energy storage systems (ESS) grows, it is crucial to valuate the technical and economic benefits of ESS deployments. Since there are many analytical tools in this space, this paper provides a review of these tools to help the audience find the proper tools for their energy storage analyses.
\end{abstract}

Recent Findings There are many software tools for valuating ESS. These tools can be classified into two groups: (1) power system simulation and planning tools for analyzing the technical contributions of ESSs, and (2) techno-economic analysis tools for valuating the economic benefits of ESS deployment and specifying the optimal design of energy systems that include ESSs. While many of the tools, developed by the national laboratories, are free to use, the commercial tools are also of great importance in meeting the customers' specific needs.

Summary This paper provides a review of software tools for ESS valuation and design. A review of analysis tools for evaluating the technical impacts of energy storage deployments is also provided, as well as a discussion of development trends for valuation and design tools.

Keywords Energy storage $\cdot$ Valuation tools $\cdot$ Analytical tools $\cdot$ Software tools

\section{Introduction}

As the electric grid evolves very quickly toward more renewable and distributed energy resources integrated with controllable loads, grid operators have experienced many technical problems in maintaining grid stability and reliability. A major concern is the high variability and uncertainty of these resources and loads [1]. Furthermore, there are challenges in mitigating the impacts of natural disasters and protecting grid infrastructure against cyber/physical threats. Energy storage systems (ESSs), with the ability to alternatively charge and discharge energy, can provide a wide range of grid services $[2,3 \bullet \bullet]$ to tackle the above challenges. There are several ways to categorize these services. A common method is based on the time scale of the charge/discharge cycle. High-power low-energy cycles

This article is part of the Topical Collection on Energy Storage

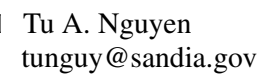

Tu A. Nguyen

tunguy@ sandia.gov

Raymond H. Byrne

rhbyrne@sandia.gov

1 Sandia National Laboratories, Albuquerque, NM 87185, USA are referred to as power applications while long-duration discharges are referred to as energy applications. Power applications transpire over short periods of time, milliseconds to seconds, and are often associated with real-time control of the power grid. Examples of power applications include frequency regulation, synthetic inertia, and small signal stability. Examples of energy applications include energy arbitrage and renewable energy time shift. Another method for classifying storage applications is assigning the benefit to generation, transmission, or distribution. It is not uncommon for a storage system to provide a benefit to more than one category. For example, a storage system is acting as a generator while discharging when it is providing a transmission or distribution deferral benefit. Another approach for categorizing storage services is by the governing rate tariff or market rules. This results in three categories: behind-the-meter (BTM) applications, front-of-the-meter (FTM) applications (e.g., market areas), and operation in a vertically integrated utility. A summary of energy storage applications is given in Table 1.

While all deployment decisions ultimately come down to some sort of benefit to cost analysis, different tools and algorithms are used to size and place energy storage in the grid depending on the application and storage operating characteristics (e.g., round-trip efficiency, life cycle). For 
Table 1 Energy storage applications

\begin{tabular}{lll}
\hline Application & Power or energy? & FTM or BTM? \\
\hline General energy applications & & \\
Energy arbitrage & Energy & FTM \\
Renewable energy firming & Energy & FTM and BTM \\
Renewable smoothing & Power & FTM and BTM \\
Ancillary services & & \\
Frequency regulation & Power & FTM \\
Operating reserve & Energy & FTM \\
Frequency response & Power & FTM \\
Voltage support & Power & FTM \\
Ramp support & Power & FTM and BTM \\
Black start & Power & FTM \\
Transmission services & & \\
Upgrade deferral & Energy & FTM \\
Congestion relief & Energy & FTM \\
Stability damping control & Power & FTM \\
Distribution services & & \\
Peak shaving & Energy & FTM and BTM \\
Voltage regulation & Power & FTM and BTM \\
Reliability and resilience & Energy and Power & FTM and BTM \\
End customers' services & & BTM \\
Time-of-use management & Energy & Power \\
Power quality & Energy & BTM \\
Resilience (back-up power) & & \\
\hline & & \\
\hline
\end{tabular}

services that provide a technical benefit, e.g., raising the frequency nadir after the loss of a large generator, dynamic simulations are required to properly size and place the energy storage system. After this analysis yields a size and location, additional analysis must be performed to compare the cost of potential alternatives. For other applications, e.g., energy arbitrage in a market, valuation analysis can provide a revenue estimate for a given storage system and a benefit/cost analysis can be easily performed. If there is a net positive benefit that meets the return on investment criteria, no further analysis is required. Therefore, as the application space for ESSs grows, energy storage valuation is of a particular interest of many energy storage stake holders (e.g., ESS owners, system operators, regulators, and researchers).

In the literature, many studies have assessed the potential revenues of ESSs for different applications such as energy arbitrage and frequency regulation in different markets [4-6], for enhancing the efficiency of current generation fleet [7], for reducing renewable energy curtailments $[8,9]$, for transmission and distribution (T\&D) upgrade deferral and congestion relief [10-12], and for BTM applications [13, 14•, 15, 16]. Taking advantages of the knowledge established in the academic literature and the expertise from the field, there are efforts from multiple parties (e.g., national laboratories, utilities, and system integrators) in developing software tools that can be used for valuing energy storage. The main purpose of such tools is to reduce the technical difficulties for the stakeholders thereby reducing the barriers to the broader deployment of ESSs. Examples of these tools include the Storage Value Estimation Tool (StorageVET) by the Electric Power Research Institute (EPRI), QuESt-Energy Storage Application Suite by Sandia National Laboratories (SNL), MASCORE-microgrid asset sizing tool by Pacific Northwest National Laboratory (PNNL), and the Renewable Energy Integration and Optimization (REopt) by the National Renewable Energy Laboratory (NREL).

Although each of the tools has its own features distinguished from others, many of them share common capabilities. In many circumstances, multiple tools must be used in order to deliver the best results. Therefore, to help the audience find the proper tools for their analyses, this paper provides (1) an overview of analytical and simulation tools used in power systems, and (2) a review of the currently available software tools for energy storage technoeconomic analyses. Since this space is growing rapidly, we also discuss the current trends in software tool developments for energy storage valuation in relation with other tools in power system field.

\section{Software Tools in Power Systems}

This section provides an overview of the different types of simulation tools required to value energy storage's technical contributions to the grid.

\section{Simulation Tools}

As with energy storage applications, there are several ways to categorize simulation tools required to value energy storage. Power system software simulation tools generally fall into one of the following categories:

- Transmission and generation modeling tools

- Distribution modeling tools

- Operation and planning tools

Each of these types of software tools is discussed in more detail in the subsequent sections.

\section{Transmission and Generation Modeling}

For energy storage applications focused on improving the dynamic performance of the grid, an electromechanical dynamic simulation tool is required to properly size and locate the energy storage so that it meets the desired 
technical performance specifications. Examples might include deploying storage to ensure that grid frequency never falls below a specified value after the loss of a large generator as well as addressing voltage issues associated with the grid integration of renewables. Transmission level modeling software packages usually include a steadystate power flow analysis tool and a dynamic simulation tool. The steady-state power flow tool can be used to perform thermal and voltage analysis, static voltage stability analysis, and transfer limit analysis. The dynamic simulation tool can be used to evaluate the dynamic response of the power system to a set of contingencies or disturbances. Prior to implementation, any control system must typically be evaluated under a wide range of operating conditions using a validated system model. The most commonly used dynamic simulation tools include General Electric's Positive Sequence Load Flow (PSLF®) [17], Siemen's PSS $®$ E [18], PowerWorld's Simulator [19], and PowerTech Labs' DSATools ${ }^{\mathrm{TM}}$ dynamic security assessment tools [20]. Both DSATools and Siemen's PSS$\mathrm{E}$ include the capability to generate a linearized state space model of the power system dynamics which is very helpful for small signal stability analysis and control system design. PowerWorld includes the capability of performing ringdown modal analysis using several popular techniques, the variable projection method (VPM) [21], the matrix pencil method [22], and dynamic mode decomposition (DMD) [23].

Hardware-in-the-loop testing is often performed to evaluate a prototype controller's interface to a model of the expected system. The two most prevalent approaches are to use a system from RTDS Technologies or OPAL-RT Technologies [24, 25]. The most prevalent software tool for

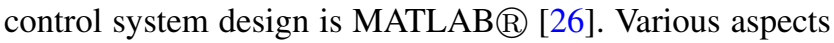
of electric power systems are easily modeled in MATLAB. A wide range of power system models are available for the MATLAB/Simulink environment. There are also several open-source MATLAB-based tools for power system design and analysis. This includes the Power System Toolbox, which is geared towards transmission level small signal and transient stability [27]. Another is the Power System Analysis Toolbox (PSAT) [28]. A comparison of PSAT and PST for high-voltage direct current (HVDC) design is found in [29].

Once these types of tools have been applied to properly size and locate an energy storage system to meet a desired technical performance specification, the next step is to apply an avoided cost analysis [30]. Using this approach, the expected costs to both the utility and society are evaluated without energy storage. Then, the same analysis is performed with the energy storage deployed in the system. For the example of meeting a frequency nadir specification after a contingency, not deploying energy storage might result in a higher probability of under-frequency load shedding and damage to equipment. Deploying energy storage might virtually eliminate these potential costs. The net benefit is then calculated by subtracting the deployment and operation costs from the expected avoided cost. The same approach can be employed to assess non-storage solutions.

\section{Distribution System Modeling}

Distribution system modeling tools provide the ability to model unbalanced power flows and faults in distribution systems. Quasi-static time series (QSTS) analysis captures time-dependent aspects of power flow, including the interaction between the daily changes in load and PV output and control actions by feeder devices, including energy storage. Energy storage deployments in a distribution grid can address technical issues related to over-/undervoltage, thermal line ratings, and excessive transformer tap changing [31]. Common distribution system modeling tools include OpenDSS [32], CYME [33], and GridLab-D [34]. Like the transmission modeling tools, these software packages can be employed to size and site energy storage in distribution networks to address technical issues. An avoided cost analysis can then be performed to identify the net benefit of deploying energy storage. Since many inverterbased distributed energy resources are being deployed in distribution networks, especially PV generation, there are often competing alternatives to deploying energy storage. All inverter-based resources are capable of providing reactive power if sized and configured properly.

\section{Operation and Planning Tools}

Production cost modeling (PCM) software is used to identify the optimal (lowest cost) dispatch of the electric power system while considering security constraints like line limits and potential contingencies. This is often referred to as security constrained economic dispatch (SCED). This economic dispatch is a key component of electricity markets as well as the operation of a vertically integrated utility. It is typically performed a day ahead and then closer to real-time (e.g., an hour ahead). The problem is usually formulated as a mixed integer linear program optimization problem. Commonly used commercial software tools include PLEXOS by Energy Exemplar [35], PROMOD by Hitachi ABB [36], Multi Area Production Simulation Software (MAPS) by General Electric [37], and GridView by Hitachi ABB [38]. In addition to these commercial PCM tools, there are also several open-source research tools developed by National Labs. They include EGRET/PRESCIENT and the Scalable Integrated Infrastructure Planning (SIIP) tool. EGRET is a Python-based 
package that provides tools for building electric grid optimization models using Pyomo [39, 40]. This software includes objects and functions for parsing, storing, and managing electric grid data. EGRET also includes the following electric grid models: ACOPF (alternating current optimal power flow); DCOPF (direct current optimized power flow) linearized transmission model; ACOPF relaxations; unit commitment; and economic dispatch [41]. SIIP is based on the mathematical programming language Julia [42]. SIIP addresses a range of quasi-static power systems scheduling problems including unit commitment and economic dispatch, automatic generation control, and nonlinear optimal power flow [43].

The expansion planning process incorporates the previously mentioned analysis tools. Based on forecasts for load growth, different scenarios can be evaluated at the transmission and distribution level to maintain the desired level of reliability at the lowest cost. While reliability was the major driver in the past, an increase in low-probability events like hurricane Maria and super storm Sandy has significantly increased interest in grid resilience. Planning approaches that properly consider resilience and reliability are now being formulated. In addition, much work still needs to be done to properly consider energy storage in expansion planning exercises.

\section{Energy Storage Valuation and Design Tools}

Techno-economic analyses of ESSs are currently focusing on two main areas:

- ESS Valuation: This is for estimating the potential benefits of an ESS providing different services. This kind of analysis is often used to find if a planned ESS deployment is financially justified or to find the maximum revenue stream of an existing ESS.
The valuation process often starts with identifying the available revenue streams for an ESS. These revenue streams will decide what storage technology will be selected. Finally, the total economic benefit is valuated given the operating characteristics of the selected technology. This step often involves an optimization problem to find the maximum revenue while considering different physical and market constraints.

- System Design: This is to find the optimal design of an integrated energy system to meet a specific design target (e.g., meeting a specific load over a period, minimizing the investment payback time of the system). The energy system can include a single ESS or multiple integrated subsystems such as roof-top PV, ESSs, on-site generators. This system is usually classified as a gridconnected or stand-alone microgrid. The optimal design often indicates the optimal size of each component of the system.

Therefore, software tools for techno-economic analyses of ESSs can also be categorized as valuation tools and design tools. Even though these tools have different features, they are often based on the optimization frameworks that find the decision variables to maximize or minimize an objective function given certain constraints. In ESS valuation tools, the optimizations are usually for finding the charge and discharge schedule of an ESS to maximize its revenue from providing certain services given its energy and power ratings and its round-trip efficiency. In system design tools, the optimization are often set up to find the optimal sizes of the system components to maximize or minimize a design objective given the system constraints.

Currently available software tools in this area are summarized in Table 2. Many of them are developed by the national laboratories. Since these tools are often funded by the U.S. Department of Energy (DOE), they are often free for the public to use. These tools are mostly developed

Table 2 Software tools for energy storage valuation and design

\begin{tabular}{|c|c|c|c|}
\hline Name & Type & Developer & Format \\
\hline \multicolumn{4}{|l|}{ Valuation Tools } \\
\hline QuESt & Free & SNL & Python-based, open-source \\
\hline StorageVET & Free & EPRI & Python-based, open-source \\
\hline energytoobase & Commercial & Energy Toolbase & Executable, Web-based \\
\hline BatSIMM & Commercial & Ascend analytics & Executable, Web-based \\
\hline \multicolumn{4}{|l|}{ Design Tools } \\
\hline MASCORE & Free & PNNL & Web-based \\
\hline MDT & Free & SNL & Executable \\
\hline DER-CAM & Free & LBNL & Executable \\
\hline REopt & Free & NREL & Web-based \\
\hline Homer & Commercial & Homer Energy & Executable, web-based \\
\hline
\end{tabular}


in Web-based (e.g., MASCORE, REopt) and open-source (e.g., StorageVET, QuESt) platforms. While a Web-based tool can be convenient to most users since no installation is required, an open-source tool can be helpful to the power users (e.g., software developers, researchers) as the tool provides access to its libraries and source code. There are also other commercial tools such as Homer [44], energy-toolbase [45], and BatterySIMM [46] developed by companies in this field. The main advantage of these tools is that they can be customized to meet the customers' specific needs. Since these commercial tools are proprietary to the paid customers, they are not reviewed in this paper.

\section{Valuation Tools}

\section{QuESt-Energy Storage Application Suite}

QuESt is an open-source software application suite for energy storage analyses developed in Python [47]. It was developed by SNL under the sponsorship of the US DOE's Energy Storage Program. It was first released in 2018 and is currently on 1.2 version. QuESt development was based upon a foundation of linear program formulations that focus on optimally valuing energy storage in market and BTM applications. QuESt consists a graphical user interface (GUI) that helps ease out the valuation process for most simple users. Since QuESt is open-source, its library that contains optimization models is also available to the users for more customized analyses and to developers for software development purposes.

QuESt's current features include QuESt Valuation for evaluating the potential revenue of an ESS participating in electricity markets, QuESt BTM for estimating cost savings for TOU and net metering customers using BTM energy storage, and QuESt Data Manager for acquiring relevant data such as electricity and service prices from ISOs and RTOs or utility rate structures. Furthermore, QuESt is also capable of implementing sensitivity analysis to facilitate planning ESS deployments. The workflow of QuESt (see Fig. 1) includes three steps: (1) decide what type of analysis,
(2) prepare the input data using QuESt Data Manager, (3) select the appropriate application to set up the analysis, run it and get the results. It is important to note that QuESt relies on Pyomo optimization modelling language that requires the installation of optimization solvers (e.g., GLPK, Gurobi). Future development of QuESt will further strengthen its current features and keep adding more capabilities such as production cost modeling, integrated resource planning (IRP), and energy storage management system (ESMS) design.

\section{StorageVET ${ }^{\mathrm{TM}}$ —Storage Value Estimation Tool}

StorageVET is a software tool developed by EPRI [48]. It is designed to help inform ESS deployment with recommended technology, location, and optimal sizes of ESSs for different grid services. Similar to QuESt, the latest version of StorageVET (ver. 2.1) is Python-based and open-source. Its current features include the evaluation of the benefits and costs of energy storage projects across different grid and customer services, the selection of storage technology, and the sensitivity analysis for optimal siting and sizing. In the future, StorageVET will be further developed into DER-VET that is a software platform to evaluate distributed energy resources (DERs).

In StorageVET, services that ESSs can provide to generate revenue are grouped into two categories:

- Pre-dispatch services include asset upgrade deferral, resource adequacy, demand response, voltage Support, backup power, and custom user constraints.

- Optimization services include retail energy time shift, retail demand charge reduction, spinning reserves, non-spinning reserves, frequency regulation, day-ahead energy time shift, and load following.

StorageVET's users first decide what kind of analysis they want to do and compose the required input data in csv format. StorageVET's Python run file is then executed to perform the analysis and record the results in the output files.
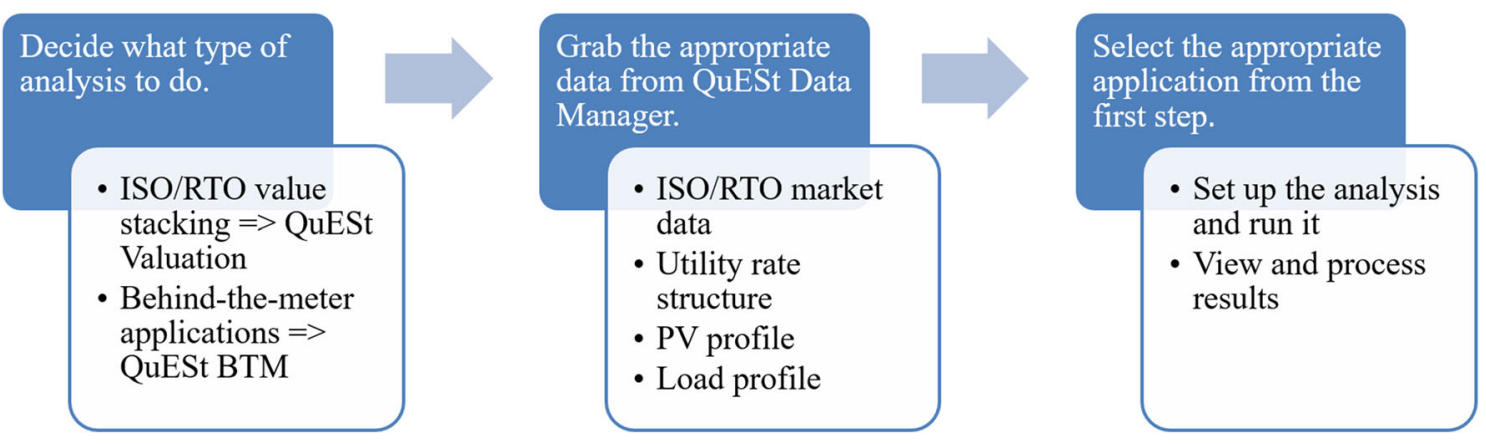

Fig. 1 QuESt's workflow 


\section{Design Tools}

\section{MASCORE—Microgrid Asset Sizing Tool}

MASCORE is a Web-based tool for microgrid asset sizing considering cost and resilience developed by PNNL [49]. The tool allows users to select, size, and operate DERs that optimize the economic performance and enhance the resilience of their microgrid systems. The tool models various DER technologies (e.g., PV, ESS, and generators) considering their underlying economic and technical characteristics. In MASCORE, the optimal sizes of different DERs are determined by solving a two-stage stochastic optimization. Since this tool is developed as a Web-based platform, the users can start their analysis right away without any installation or input data preparation. This helps provide quick solutions to most simple users. Future development of MASCORE will focus on improving DER models and optimization methods for microgrid evaluation and design.

\section{MDT—Microgrid Design Toolkit}

The Microgrid Design Toolkit (MDT), developed by SNL, is a decision support software tool for microgrid design [50]. The tool uses search algorithms such as genetic algorithms to find and evaluate different microgrid designs that meet user-defined objectives such as cost, performance, and reliability. MDT allows users to effectively investigate a large design space and find the trade-offs between design decisions. Current capabilities of MDT include topology and asset selection optimization, multi-objective optimization for search space exploration, energy and asset reliability calculations, and performance statistics generated for microgrid designs.

MDT is freely available and currently on version 1.2. MDT's users will need to install the software and run the executable file to start the program. Users can enter input data and configure other parameters for their analyses. MDT's output features allow users to explore the design space and extract the results.

\section{DER-CAM—Distributed Energy Resources Customer Adoption Model}

DER-CAM is a decision support tool, developed by Lawrence Berkeley National Laboratory (LBNL), to find the optimal investments on new DERs for buildings or microgrids [51]. DER-CAM's users can set up an analysis as single-objective or multi-objective optimization. A common problem that can be solved by DER-CAM is optimizing the DER investments to minimize the total annual energy supply cost of a building or a microgrid. DER-CAM also provides optimal dispatch of DERs participating in energy markets. Current capabilities of DER-CAM include optimizing portfolio of DER to meet a certain objective, optimal siting and sizing DERs to minimize costs, and optimal dispatching DERs to maximize customer benefits.

Like many other tools developed by the national laboratories, DER-CAM is free. To use the software, the users will need to download and install a user interface application. The workflow of DER-CAM includes several steps: (1) define topology or select single-node model, (2) define load and other site data, (3) define tariff structures, (4) define DER parameters, (5) run the base-case without new DERs, (6) define investment options for new DERs, and (7) run the optimization to find optimal DER sizes.

\section{REopt-Renewable Energy Integration and Optimization}

REopt is a software tool, developed by NREL, to optimize the integration and operation of energy systems for buildings, campuses, communities, and microgrids [52]. REopt capability is based upon an optimization that is formulated as a mixed-integer linear program to find the optimal mix, sizes, and dispatch of different DERs and loads to minimize cost. REopt includes a wide range of technologies of DERs and loads such as PV, energy storage, wind, biomass, waste energy, combustion turbines, diesel and natural gas generators, solar water heating, solar ventilation air preheating, ground source heat pumps, and controllable loads.

Currently, REopt includes a Web-based version (REopt Lite) that is free to the public to use. The workflow of REopt Lite includes the following steps: (1) gather data, (2) select either financial or resilience focus, (3) define site data (location, load profile, tariff structures), (4) select DER technologies (PV, wind, storage) and specify their parameters, (5) run the tool and get results. The users can also contact the REopt team for more customized analyses that require the full capabilities of REopt.

\section{Conclusions, Gaps, and Trends}

As of today, even though many tools for ESS valuation and design exist, there is a lack of integrated tools that are capable of both analyzing the technical performance and valuating the economic benefits of ESSs. Most technoeconomic tools ignore the underlying characteristics of ESS such as state of health and non-linear operating behaviors. Furthermore, as the application space of energy storage grows very quickly across the entire grid from generation, transmission, distribution to load, the tools are also required to analyze ESSs' interoperability across 
different spaces (e.g., ESSs that are located in distribution systems but provide transmission services). Therefore, development efforts in this area are trying to fill these gaps. Specifically, current development trends focus on three main areas: (1) developing more accurate energy storage models that can capture the non-linear degradation and operating characteristics of different storage technologies, (2) incorporating these ESS models in existing simulation and techno-economic tools, (3) integrating multiple tools in one platform to provide better capability to the users. While many of the tools, developed by the national laboratories, are free to use, the commercial tools are also of great importance in meeting the customers' specific needs. In the near future, these development trends are still of great interest to all stakeholders until the gaps are filled.

Acknowledgements Sandia National Laboratories is a multimission laboratory managed and operated by National Technology \& Engineering Solutions of Sandia, LLC, a wholly owned subsidiary of Honeywell International Inc., for the U.S. Department of Energy's National Nuclear Security Administration under contract DE-NA0003525.

Funding This work was funded by the U.S. Department of Energy Office of Electricity Energy Storage Program under the guidance of Dr. Imre Gyuk.

\section{Declarations}

Disclaimer This paper describes objective technical results and analysis. Any subjective views or opinions that might be expressed in the paper do not necessarily represent the views of the U.S. Department of Energy or the United States Government.

Human and Animal Rights and Informed Consent This article does not contain any studies with human or animal subjects performed by the authors.

Conflict of interest The authors declare that they have no conflict of interest.

Open Access This article is licensed under a Creative Commons Attribution 4.0 International License, which permits use, sharing, adaptation, distribution and reproduction in any medium or format, as long as you give appropriate credit to the original author(s) and the source, provide a link to the Creative Commons licence, and indicate if changes were made. The images or other third party material in this article are included in the article's Creative Commons licence, unless indicated otherwise in a credit line to the material. If material is not included in the article's Creative Commons licence and your intended use is not permitted by statutory regulation or exceeds the permitted use, you will need to obtain permission directly from the copyright holder. To view a copy of this licence, visit http://creativecommons. org/licenses/by/4.0/.

\section{References}

Papers of particular interest, published recently, have been highlighted as:

- Of importance

$\bullet$ Of major importance

1. Manz D, Walling R, Miller N, LaRose B, D'Aquila $\mathrm{R}$, Daryanian B. The grid of the future: ten trends that will shape the grid over the next decade. IEEE Power Energy Mag. 2014;12(3):26-36.

2. Eyer J, Corey G. Energy storage for the electricity grid: benefits and market potential assessment guide. Tech. rep., Sandia National Laboratories, Albuquerque, NM SAND20100815. 2010.

3.• Byrne R, Nguyen T, Copp DA, Chalamala BR, Gyuk I. Energy management and optimization methods for grid energy storage systems. IEEE Access. 2018;6:13231-60. The paper presents a comprehensive review of the applications of energy storage as well as the optimization methods used in evaluating energy storage technical and economic benefits. Many of the software tools for energy storage valuation and design are based on the optimization methods reviewed in this paper.

4. Nguyen T, Copp DA, Byrne R, Chalamala BR. Market evaluation of energy storage systems incorporating technologyspecific nonlinear models. IEEE Trans Power Syst. 2019;34 (5):3706-15.

5. Tian Y, Bera A, Benidris M, Mitra J. Stacked revenue and technical benefits of a grid-connected energy storage system. IEEE Trans Ind Appl. 2018;54(4):3034-43.

6. Concepcion RJ, Wilches-Bernal F, Byrne R. Revenue opportunities for electric storage resources in the southwest power pool integrated marketplace. In: Proceedings of the 2019 IEEE power and energy society general meeting (PESGM). Atlanta; 2019. p. 1-5.

7. Nguyen T, Byrne R, Trevizan RD. Utilization of existing generation fleets using large-scale energy storage systems. In: Proceedings of the 2020 IEEE power energy society general meeting. Montreal; 2020. p. 1-5.

8. Guo Z, Wei W, Chen L, Dong Z, Mei S. Impact of energy storage on renewable energy utilization: a geometric description. IEEE Trans Sustain Energy. 2020;1-1.

9. Bitaraf H, Rahman S. Reducing curtailed wind energy through energy storage and demand response. IEEE Trans Sustain Energy. 2018;9(1):228-36.

10. Arteaga J, Zareipour H, Amjady N. Energy storage as a service: optimal pricing for transmission congestion relief. IEEE Open Access J Power Energy. 2020;1-1.

11. Deeba SR, Sharma R, Saha TK, Chakraborty D, Thomas A. Evaluation of technical and financial benefits of battery-based energy storage systems in distribution networks. IET Renew Power Gener. 2016;10(8):1149-60.

12. Khani H, Zadeh MRD, Hajimiragha AH. Transmission congestion relief using privately owned large-scale energy storage systems in a competitive electricity market. IEEE Trans Power Syst. 2016;31(2):1449-58.

13. Neubauer J, Simpson M. Deployment of behind-the-meter energy storage for demand charge reduction. 2015.

14. Wu D, Kintner-Meyer M, Yang T, Balducci P. Economic analysis and optimal sizing for behind-the-meter battery storage. In: 2016 IEEE power and energy society general meeting (PESGM); 2016. p. 1-5. This paper presents the 
valuation and sizing of energy storage for behind-the-meter application that is the focus of many tools.

15. Nguyen T, Byrne R. Maximizing the cost-savings for time-ofuse and net-metering customers using behind-the-meter energy storage systems. In: Proceedings of the 2017 North American power symposium (NAPS). Morgantown; 2017. p. 1-5.

16. Nguyen T, Byrne R. Optimal time-of-use management with power factor correction using behind-the-meter energy storage systems. In: Proceedings of the 2018 IEEE power energy society general meeting. Portland; 2018. p. 1-5.

17. Positive Sequence Load Flow (PSLF) Software. General ELectric Corporation (2020).

18. PSS/E. Siemens (2020).

19. PowerWorld Simulator. PowerWorld Corporation (2020).

20. DSATools, dynamic security assessment tools. Powertech Labs Inc (2020).

21. Borden AR, Lesieutre BC. Variable projection method for power system modal identification. IEEE Trans Power Syst. 2014;29(6):2613-20. https://doi.org/10.1109/TPWRS.2014.23 09635.

22. Grant LL, Crow ML. Comparison of matrix pencil and prony methods for power system modal analysis of noisy signals. In: 2011 North American power symposium; 2011. p. 1-7. https://doi.org/10.1109/NAPS.2011.6024892.

23. Trinh W, Overbye T. Comparison of dynamic mode decompositon and iterative matrix pencil method for power system modal analysis. In: 2019 International conference on smart grid synchronized measurements and analytics (SGSMA); 2019. p. 1-6. https://doi.org/10.1109/SGSMA.2019.8784536.

24. RTDS Simulator. RTDS Technologies (2020).

25. OPAL-RT Real-time digital simulator. OPAL-RT Technologies (2020).

26. MATLAB: version R2020b. The MathWorks Inc., Natick, Massachusetts (2020).

27. Chow JH, Cheung KW, Rogers G. Power System Toolbox, Version 3.0. 2020.

28. Milano F. Power System Analysis Toolbox, Version 2.1.11. 2020.

29. Ubisse AV, Folly KA, Awodele KO, Oyedokun DT. Comparison of MATLAB PST, PSAT and DigSILENT for transient stability studies on parallel HVACHVDC transmission lines. In: 45th International universities power engineering conference UPEC2010; 2010. p. 1-6.

30. Beecher JA. Avoided cost: an essential concept for integrated resource planning. J Contemp Water Res Educ. 1996;104:2835.

31. Reno MJ, Lave M, Quiroz JE, Broderick RJ. Pv ramp rate smoothing using energy storage to mitigate increased voltage regulator tapping. In: 2016 IEEE 43rd photovoltaic specialists conference (PVSC); 2016. p. 2015-2020. https://doi.org/10. 1109/PVSC.2016.7749982.

32. Dugan RC, McDermott TE. An open source platform for collaborating on smart grid research. In: 2011 IEEE power and energy society general meeting; 2011. p. 1-7. https://doi.org/10. 1109/PES.2011.6039829.
33. CYME Power Engineering Software. Easton Corporation Plc, Dublin, Ireland (2020).

34. Chassin DP, Schneider K, Gerkensmeyer C. Gridlab-d: an open-source power systems modeling and simulation environment. In: 2008 IEEE/PES transmission and distribution conference and exposition; 2008. p. 1-5. https://doi.org/10.1109/TDC. 2008.4517260.

35. Nweke CI, Leanez F, Drayton GR, Kolhe M. Benefits of chronological optimization in capacity planning for electricity markets. In: 2012 IEEE international conference on power system technology (POWERCON); 2012. p. 1-6. https://doi.org/ 10.1109/PowerCon.2012.6401421.

36. Quinn P, Thappetaobula R. Transmission portfolio screening using promod analysis tool (august 2009). In: IEEE PES T D 2010; 2010. p. 1-5. https://doi.org/10.1109/TDC.2010.5484 555.

37. General Electric (GE) Multi Area Production Simulation Software program (MAPS). General Electric Energy Consulting (2020).

38. Feng X, Tang L, Wang Z, Yang J, Wong W, Chao H, Mukerji R. A new breed of software tool for integrated electrical power system and market analysis-gridview. In: IEEE Power engineering society summer meeting, vol. 2; 2002. p. 737-43. https://doi.org/10.1109/PESS.2002.1043404.

39. Hart WE, Laird CD, Watson JP, Woodruff DL, Hackebeil GA, Nicholson BL, Siirola JD. Pyomo-optimization modeling in python, vol. 67, 2nd edn. Springer Science \& Business Media. 2017.

40. Hart WE, Watson JP, Woodruff DL. Pyomo: modeling and solving mathematical programs in python. Math Program Comput. 2011;3(3):219-60.

41. General Electric (GE) Multi Area Production Simulation Software program (MAPS). Sandia National Laboratories (2020).

42. Bezanson J, Edelman A, Karpinski S, Shah VB. Julia: a fresh approach to numerical computing. SIAM Rev. 2017;59(1):6598. https://doi.org/10.1137/141000671.

43. Scalable Integrated Infrastructure Planning (SIIP) Model. National Renewable Engineering Laboratory (NREL), Golden, Colorado (2020).

44. Homer Energy. https://www.homerenergy.com/.

45. Energy Toolbase. https://www.energytoolbase.com.

46. BatterySIMM Suite. https://www.ascendanalytics.com/solutions/ batterysimm-suite.

47. QuESt v1.2. https://energy.sandia.gov/sandia-releases-quest-v1-2.

48. StorageVET v2.1. https://www.storagevet.com.

49. Energy storage valuation tool. https://eset.pnnl.gov.

50. Microgrid Design Toolkit (MDT). https://www.sandia.gov/ CSR/tools/mdt.html.

51. The Distributed Energy Resources Customer Adoption Model (DER-CAM). https://gridintegration.lbl.gov/der-cam.

52. REopt: Renewable Energy Integration and Optimization. https://reopt.nrel.gov/tool.

Publisher's Note Springer Nature remains neutral with regard to jurisdictional claims in published maps and institutional affiliations. 